Meta

Journal des traducteurs

Translators' Journal

\title{
Nos langues : structures instrumentales, structures mentales
}

\section{Mario Wandruszka}

Volume 16, numéro 1-2, mars 1971

Actes du colloque international de linguistique et de traduction.

Montréal, 30 septembre - 3 octobre 1970

URI : https://id.erudit.org/iderudit/004590ar

DOI : https://doi.org/10.7202/004590ar

Aller au sommaire du numéro

Éditeur(s)

Les Presses de l'Université de Montréal

ISSN

0026-0452 (imprimé)

1492-1421 (numérique)

Découvrir la revue

Citer cet article

Wandruszka, M. (1971). Nos langues : structures instrumentales, structures mentales. Meta, 16(1-2), 7-16. https://doi.org/10.7202/004590ar d'utilisation que vous pouvez consulter en ligne. 


\section{Nos langues: structures instrumentales, structures mentales}

La linguistique, elle aussi, est aujourd'hui en pleine révolution. Dans un joyeux tumulte d'idées, de doctrines, d'hypothèses sans cesse remaniées, repensées, remises en question, une nouvelle théorie du langage humain est en train de s'élaborer. Il est encore trop tôt pour savoir dans quelle mesure ce nouveau modèle théorique du langage pourra un jour nous aider à mieux comprendre la traduction en tant que phénomène profondément humain, la nature, les possibilités et les limites du bilinguisme individuel sur lequel repose, en dernière analyse, la traduction; dans quelle mesure une linguistique nouvelle nous permettra de mieux saisir les problèmes à la fois si complexes et si subtils du noble art de la traduction. Mais d'ores et déjà, avertie par la série des brillants échecs qui, pendant vingt-cinq ans, ont jalonné la route de la traduction automatique, cette nouvelle linguistique peut nous fournir une analyse plus exacte des principales qualités qui, à la différence des langues «artificielles», des langues «construites» pour ordinateurs, caractérisent nos langues dites « historiques» ou «naturelles». Les propriétés spécifiques de nos langues naturelles peuvent, en effet, se résumer ainsi : elles sont faites $1^{\circ}$ d'analogies et d'anomalies, $2^{\circ}$ de polymorphies et de polysémies, $3^{\circ}$ de redondances et de déficiences, $4^{\circ}$ d'explicitations et d'implications, $5^{\circ}$ de constantes et de variantes.

Or, ce sont précisément ces propriétés-là qui se manifestent dans chaque phrase, chaque mot d'une traduction, propriétés qui font le bonheur et le désespoir du traducteur!

\section{I}

Nos langues sont faites de systèmes analogiques et d'anomalies asystématiques; de règles, d'exceptions aux règles et d'exceptions aux exceptions. Rien de plus familier au traducteur que ces anomalies instrumentales différant d'une façon parfois si bizarre d'une langue à l'autre. Si au pluriel systématique anglais the peoples (The peoples of the world, the peoples of Asia) correspond le pluriel français les peuples, le traducteur doit savoir que le pluriel asystématique sans $s$ : people, qui a fini par assumer des fonctions assez différentes, est en fait infiniment plus fréquent. Dans les phrases suivantes (Margaret Mitchell, Gone with the Wind, p. 78, 470, 128) : "Love isn't enough to make a successful 
marriage when two people are as different as we are», "There were fifty or sixty people there», "Do you care if people talk?», le traducteur français a rendu two people par deux êtres, fifty or sixty people par cinquante à soixante personnes, people par les gens. Le traducteur connaîtra bien entendu toutes les anomalies de ce pluriel masculin les gens qui n'a pas de singulier (sauf pour les gendarmes, qui se sont créé leur propre singulier systématique, le gendarme); il sait qu'aux gens de lettres correspond au singulier l'homme de lettres, et non pas le gendelettre comme on a dit parfois plaisamment; il sait que les gens sont du masculin, mais qu'il y a là aussi des anomalies : les bonnes gens, les vieilles gens; que le singulier la gent n'est plus qu'un vague souvenir littéraire, évoquant la gent trotte-menu, la gent marécageuse de La Fontaine, et que par conséquent, si en italien la gente, en espagnol la gente, en portugais a gente appartiennent aujourd'hui à la langue la plus quotidiennement familière, le traducteur français ne saurait rendre ces mots en se servant de leur équivalent formel français, la gent! Le caractère fortuit, accidentel, capricieux de toutes ces anomalies asystématiques inscrites dans chacune de nos langues à des endroits différents devient plus sensible encore dans cette confrontation de deux langues qu'est la traduction.

\section{II}

Nos langues naturelles sont parsemées de polymorphies et de polysémies. Les rapports entre les formes et leurs fonctions ne sont pas ce qu'ils devraient être dans un système d'information construit avec la rigueur propre aux formules mathématiques. À chaque instant, nous voici devant une polymorphie, devant plusieurs formes qui s'offrent à nous pour remplir plus ou moins la même fonction. Le traducteur le sait bien, puisque sans cesse il doit choisir : traduira-t-il dans un texte donné the second par le deuxième ou par le second; I can par je peux ou par je puis; did you see him par l'as-tu vu, est-ce que tu l'as vu ou tu l'as vu...; impatiently par impatiemment ou avec impatience; arriving par arrivant ou bien par qui arrive? Inversement, le traducteur pourra, et devra choisir, pour le voisinage : the neighborhood ou the vicinity; pour la solitude : loneliness ou solitude; pour la liberté : freedom ou liberty; pour merveilleux : wonderful ou marvelous; pour inévitable : unavoidable ou inevitable; pour permettre : to allow ou to permit ; pour répondre : to answer ou to reply...

Le résultat de cette polymorphie est la synonymie, partielle et dans certains cas même totale, entre plusieurs formes pour une seule fonction, et cette concurrence synonymique est créatrice de disponibilités stylistiques, permettant ainsi de choisir les connotations les plus nuancées et les plus délicates.

La polysémie, au contraire, confie plusieurs fonctions à une seule forme. Le passage peut signifier l'action de passer, mais aussi l'endroit par où l'on passe (le passage souterrain, le passage à niveau), ou cncore une suite de phrases, de mots dans un texte (relire tout le passage). La marche peut être l'action de marcher, d'un homme, d'une troupe, d'un train, d'un moteur; la progression des événements, des affaires; une pièce de musique ; un des éléments de construction dont se compose un escalier. Le cours, la course nous offrent des polysémies 
tout aussi variées. Or, cette pluralité de fonctions pour une seule forme varie très souvent d'une langue à l'autre : le passage, la marche, le cours, la course ne peuvent pas être traduits toujours par the passage, the march, the course, il s'en faut de beaucoup ; dans ces deux langues, des mots formellement correspondants ont souvent développé des polysémies curieusement différentes. Beaucoup de «faux amis», ces bêtes noires du traducteur, résultent ainsi des caprices de la polysémie. Il suffit de rappeler ici la place, the place; l'issue, the issue; l'engin, the engine; l'essence, the essence; la lecture, the lecture; le papier, the paper ; la figure, the figure; le dessein, le dessin, the design... (ce mot qui dernièrement a pris encore une acception nouvelle et qui, dans cet emploi, est en passe de devenir un mot français : voir l'Express du 20 juillet 1970 , p. 58 : « Le design. Tout le monde en parle. Mais que recouvre ce mot un peu barbare qui ne possède pas d'équivalent en français? »).

De toutes les propriétés spécifiques de nos langues naturelles, la polysémie est peut-être la plus étonnante. Elle nous permet de confier à une forme donnée des fonctions nouvelles à côté de celles déjà existantes. Le satellite, en latin le garde du corps d'un homme influent, en français un corps céleste tournant autour d'un autre, s'est enrichi de nos jours de deux emplois nouveaux : nous nous servons de ce mot pour désigner soit un engin de télécommunication, soit un État gravitant autour d'un autre plus puissant (métaphore toujours un peu péjorative : les satellites de Moscou).

Avec quelle facilité le mot espace s'est-il adjugé une signification nouvelle, sans pour autant perdre les anciennes! Il y a quarante ans, personne n'aurait su deviner ce que pouvait bien être des rescapés de l'espace! Qui, en 1930, aurait su prévoir qu'en 1970 un cadre serait non seulement l'objet servant à encadrer un tableau, mais aussi un homme qui, dans une entreprise industrielle, exerce des fonctions de responsabilité moyenne et supérieure, qui appartient donc aux cadres de l'entreprise tout comme on pouvait appartenir, depuis la Révolution française, aux cadres de l'armée ${ }^{1}$ ? C'est grâce aux différents procédés d'enrichissement polysémique que l'entreprise (ce que l'on entreprend) en est venue à signifier aussi une firme industrielle, que l'ensemble peut faire penser à des acteurs, à des musiciens, mais aussi aux ensembles de la mode féminine, à des ensembles industriels, médicaux, scolaires, urbains, à la théorie des ensembles des mathématiciens...

Ces quelques exemples nous permettent de mesurer l'importance de la polysémie, la flexibilité, l'élasticité qu'elle confère à nos langues naturelles. Pour les objets nouveaux, les idées nouvelles qui, aujourd'hui, en nombre sans cesse croissant et à une allure de plus en plus vertigineuse, transforment notre univers, point n'est besoin de créer chaque fois un mot inédit. Souvent, la polysémie nous permet de mettre le vin nouveau dans les vieilles outres. Rappelons-nous les fonctions nouvelles confiées depuis peu à certains mots anglais comme stress, image, status, establishment, environment...

L'extension polysémique est parfois à peine perceptible; elle peut par là

1. Jean Darbelnet, "Mots nouveaux et sens nouveaux ", Journal des traducteurs, IX, 2 (1964), p. 42. 
même présenter un problème particulièrement délicat au traducteur. Voici un exemple : dans son livre The New Europeans (Londres, 1968, p. 271), Anthony Sampson, parlant des différentes langues parlées en Europe, s'interroge : «Who are the best and worst linguists? 》 La première surprise passée, nous comprenons sans difficulté que l'auteur ne pense nullement aux mérites respectifs des grands maîtres, des équipes de chercheurs de la linguistique contemporaine, mais à la disposition et à l'aptitude des différents peuples à apprendre des langues étrangères, car il poursuit : "My own random observation would put Americans well at the bottom, closely followed by the Spanish and French, with the British and Italians not far ahead...» Voici comment cette question a été traduite dans l'édition française de ce livre : "Quels sont les linguistes les plus doués»? En anglais, l'extension polysémique : a linguist $=$ un connaisseur, un amateur de langues étrangères, est chose assez courante déjà ; en français, elle choque encore un peu par sa nouveauté.

Polymorphie et polysémie : deux phénomènes complémentaires, deux facteurs décisifs pour donner à nos langues leur inégalable souplesse, cette disponibilité des formes qui est l'une des conditions, et pas des moindres, de ce qu'on est convenu d'appeler la créativité du langage humain.

III

Nos langues sont pleines de redondances, d'informations données plusieurs fois dans un même énoncé, et de déficiences, d'informations incomplètes et par conséquent ambiguës. Dans le titre du livre d'Anthony Sampson, The New Europeans, seul l's final du substantif nous dit que nous devons penser aux européens au pluriel. Le titre français du même ouvrage fait entendre cette information (pluriel) deux fois, le titre italien trois fois :

angl. The New Europeans

fr. Les nouveaux européens

[द] [oz]

it. I nuovi europei

[i] [i] [i]

L's final du pluriel s'étant amuï, quand il n'était pas maintenu par la liaison, c'est le plus souvent l'article qui, en français, doit nous dire si le nom en question est un singulier ou un pluriel :

angl. The political parties

fr. Les partis politiques

[డ]

it. $\quad I$ partiti politici

[i] [i] [tchì]

Mais les pluriels irréguliers des substantifs nous donnent également, dans leur forme sonore, cette information; jointe à celle contenue dans l'article, il en résulte une information redondante :

le parti - les partis - le journal - les journaux

$\left[\right.$ [ə] ${ }_{[\epsilon]}[]_{[\epsilon]}[0]$ 
D'une langue à l'autre, les redondances sont réparties de façon différente. En français, dès que l'information contenue dans le contexte exclut l'équivoque, le pronom possessif peut être remplacé par l'article défini : les mains dans les poches; en anglais, il faut presque toujours répéter le pronom : his hands in his pockets. L'information déficiente, l'ambiguïté peut être le résultat d'une structure instrumentale insuffisamment différenciée. L'anglais distingue dans ses pronoms possessifs le sexe de la personne qui possède : his mother, her mother. Le français n'a qu'une seule forme : sa mère. Cela peut créer une ambiguité ; pour la résoudre, on peut avoir recours au pronom personnel : sa mère à lui, sa mère à elle. La réparation d'une déficience peut ainsi aboutir à une redondance.

L'ambiguïté se mesure en fonction de l'importance de l'intérêt que possède l'élément d'information manquant. L'absence de différenciation sexuelle dans la plupart des mots anglais peut conduire à des ambiguïtés parfois assez troublantes ( $a$ friend $=$ un ami, ou une amie) et exiger un supplément d'information (boy friend, girl friend, woman friend, etc.). Le titre du best-seller caricatural de Penelope Ashe, Naked Came the Stranger (New York, 1969) ne nous dit pas si nous devons penser à un étranger ou à une étrangère. Une photo sur la couverture du livre nous donne, il est vrai, ce supplément d'information qui, dans ce cas, n'est pas sans intérêt. La langue française est ici plus explicite. Voici le titre de la version française qui vient de paraître : l'Étrangère est arrivée nue.

Aujourd'hui les linguistes de toutes tendances portent un intérêt tout particulier à ces deux phénomènes complémentaires de la redondance et de l'ambiguïté, qui pour le traducteur sont une expérience quotidienne.

\section{IV}

Nos langues sont faites d'explicitations et d'implications. Qu'on nous parle de la terre des hommes et nous comprenons sans qu'on nous le dise expressément que ce sont tous les êtres humains qui sont ici visés, hommes, femmes et enfants, tandis que dans nos grands magasins, les rayons pour hommes s'opposent à d'autres rayons destinés aux femmes, aux enfants. Nous savons quel sens il faut donner chaque fois à un mot aussi polysémique parce qu'il ne nous est pas présenté isolément : il est entouré d'un contexte linguistique, il baigne dans une situation extralinguistique qui orientent notre choix. Sans cette orientation fournie par la situation et le contexte, la polysémie, l'un des grands moyens d'assouplissement et d'enrichissement de nos langues, nous serait rigoureusement interdite, car elle nous conduirait à des ambiguïtés sans fin. C'est grâce à cette orientation que nous comprenons que tel routier est un conducteur de poids lourds effectuant de longs trajets sur route, tel autre un coureur cycliste sur route; que ce chemisier est un fabricant ou vendeur de chemises, et cet autre chemisier une blouse féminine de coupe masculine; que suivant la situation et le contexte un bananier est un arbre qui porte des bananes ou un cargo qui les transporte, un pétrolier un navire citerne ou un industriel qui s'occupe de pétroles, un glacier un amas de glaces ou un fabricant ou vendeur de glaces... Le dosage des explicitations et des implications peut varier d'une structure à une autre et d'une langue à l'autre. L'implication est plus grande dans un mot français formé à l'aide de la désinence 
-ier, désinence très polysémique, que dans le mot composé correspondant de l'anglais. Apple-tree, wasps' nest, goatherd, milkman, ceiling lamp, ink-pot, ashtray, salad-bowl, chess-board, sont plus explicites que pommier, guêpier, chevrier, laitier, plafonnier, encrier, cendrier, saladier, échiquier...

Le problème du dosage des explicitations et des implications se pose pour chaque phrase que nous prononçons, que nous écrivons. Il se pose à nouveau au traducteur. Souvenons-nous des analyses magistrales de MM. Vinay et Darbelnet à ce sujet. En général, pour les raisons les plus diverses, le texte de la traduction a tendance à être plus explicite que celui de l'original. Voici quelques exemples pris dans des articles parus en anglais dans le Reader's Digest (juillet 1970 ) et dans la Sélection du Reader's Digest du même mois : «A 1969 report of the Royal Commission on Security» (p. 33), «Un rapport de la Commission royale sur la sécurité, daté de 1969 ; "In 1960, Bourassa came home from Oxford and Harvard》 (p. 83), "En 1960, ses études terminées à Oxford et à Harvard, Bourassa revint au Canada ; «at a delirious Liberal victory gathering 》 (p. 85), "au cours de la réunion enthousiaste célébrant la victoire des libéraux »; *In our laboratory, in fact, the sustained stress of a screeching siren was enough to kill rats 》 (p. 116), «Dans notre laboratoire, le stress prolongé causé par le hurlement d'une sirène a été suffisant pour tuer des rats ».

Bien entendu, le phénomène contraire peut se produire également. La traduction est moins explicite que l'original dans les exemples suivants : « a blockby-block surveillance of an area under suspicion» (p. 31), «la surveillance étroite d'un quartier suspect»; "Bleeding badly, the wounded Johnstone pulled himself up to return fire. He shot one bandit in the shoulder, spinning him backward and knocking the gun out of his hand》 (p. 34), "Ruisselant de sang, il se redresse, tire à son tour. L'un des malfaiteurs, atteint à l'épaule, tombe à la renverse et lâche son arme ». Le groupe verbal anglais, composé d'un verbe et d'une particule verbale ou encore d'autres compléments adverbiaux, favorise l'explicitation : to pull oneself up est sans doute plus explicite que se redresser, to knock out of one's hand plus expressif que faire lâcher. Assez souvent, le traducteur français doit renoncer à trouver à un groupe verbal de ce genre, un équivalent possédant le même degré d'explicitation. Voici le sous-titre qui orne le récit de cet exploit policier : "Outgunned but not outfought». Nous imaginons sans peine les efforts désespérés du traducteur français avant de se rendre à l'évidence de leur inutilité et de se contenter d'un sous-titre quelconque qui n'évoque que de très loin, il faut l'avouer, l'original : «Sens du devoir »!

$\mathrm{V}$

Chacune de nos langues est composée de constantes et de variantes. Le jeu des variantes, nous le savons, est d'une complexité extraordinaire : variantes dialectales, régionales, locales, variantes sociales et culturelles, que les lexicographes s'efforcent de démêler en indiquant pour certains mots le registre particulier auquel ils appartiennent : registre familier, populaire, vulgaire, argotique, poétique, littéraire..., variantes historiques, enfin, par rapport à l'état présent de la langue, archaïsmes qui ne sont plus des constantes de cette langue, néologismes qui ne le: sont pas encore... 
C'est sans doute le mélange incessant de toutes ces variantes, l'incomparable variété de leur dosage, qui opposent le plus fortement nos langues naturelles à toute langue construite artificiellement; c'est sans doute là que le traducteur se heurte aux obstacles les plus sérieux, que les limites de toute traduction nous apparaissent le plus clairement. D'une langue à l'autre, les variantes sont réparties différemment. La langue $A$ peut disposer d'une variante à tel ou tel point précis de ses structures phonétiques, lexicales, syntaxiques, là où la langue $B$ ne possède absolument rien. Exemple : en anglais, les pronoms thou, thee, thy sont aujourd'hui des archaïsmes, des variantes bibliques et littéraires pour you et your; un auteur contemporain peut donner à sa phrase une certaine couleur archaïque, pathétique, ironique, rien qu'en y insérant une de ces formes. Pour rendre cette nuance, le traducteur français ne trouve rien d'équivalent dans ses structures pronominales, toi et ton appartenant en français aux constantes au même titre que vous et votre; le seul moyen qui lui reste est de chercher à placer un archaïsme lexical français à un autre endroit de cette phrase, pour lui donner une tonalité semblable.

Les variantes dialectales, régionales, locales, sociales présentent sans cesse les mêmes difficultés. Dans le roman de Gabrielle Roy Bonheur d'accasion, la famille Lacasse, du faubourg Saint-Henri à Montréal, a conservé la vieille formule de respect paysanne son père, sa mère, son oncle, sa tante («Écoute, sa mère, $j$ 'ai quelque chose à te dire. C'est pas de son père que je voulais te parler... », nouvelle édition, Montréal, 1967, p. 62). Dans la traduction anglaise (The Tin Flute, traduit du français par Hannah Josephson, New Canadian Library $\mathrm{n}^{\circ}$ 5, Toronto et Montréal), son père et sa mère deviennent tout simplement Father et Mother : un trait caractéristique de la psychosociologie de cette famille a disparu. In en va de même de presque toutes les autres variantes canadiennesfrançaises qui caractérisent le discours des gens de Saint-Henri, variantes par rapport à la langue de la narratrice, qui est la langue française littéraire du $\mathrm{XX}^{\mathrm{e}}$ siècle. Les nombreux mots en -eux, en français, de savoureuses variantes populaires pour autant de substantifs-adjectifs en -eur, apparaissent dans la traduction anglaise sans ce coefficient sociologique : les chômeux (p. 54), the unemployed; les quêteux (p. 56), beggars; des penseux (p. 56), thinkers; leurs beaux parleux (p. 48), their fine talkers; not grand parleur et petit faiseux (p. 130), our spellbinder!

Insérées dans un récit français toutes ces variantes canadiennes-françaises, tous les canadianismes, québécismes, montréalismes, ont une valeur évocatrice particulière. Ainsi, pour citer un dernier exemple, jongler pour « réfléchir, faire des projets, se faire des soucis, tourner et retourner des idées dans sa tête »: "Qu'est-ce que t'as à jongler, sa mère? » (p. 155), «Now what's on your mind, Mother?» "Ben voyons sa mère, autant être assis icitte comme ailleurs, tant qu'à être assis pour jongler.» «Jongler! T'es-tu pas capable de faire autre chose? T'as passé quasiment toute ta vie à jongler. Et au bout de toutes tes jongleries, t'as jamais été plus avancée » (p. 80), «Now look here, Mother, I might as well sit here as anywhere else. I might as well be seated while? Make my plans.» «Make plans! Can't you do anything else? All your life you've been making plans. And where did it get you? You're no further ahead.» «Tu dors pas, hein? ? « Non. » « Tu jongles, toi aussi ?... Jongle pas trop. Ça donne rien... 
Des jongleries c'est pas ça qui aide » (p. 250), «Can't you sleep?» «No.» «Can't you stop fretting?... Don't wrack your brains too much. It does no good... It doesn't help to wrack your brains. »

Dans la traduction anglaise, on le voit, la connotation sociologique particulière a disparu; to make plans, to fret, to wrack one's brains : la variante canadienne-française est rendue par des constantes anglaises. Bien sûr la traductrice aurait pu chercher des équivalents dans l'inépuisable slang anglo-saxon. Mais dans quelle variété du slang britannique ou américain aurait-elle pu transposer ce jongler sans introduire une fausse note dans ces rapports, somme toute intraduisibles, entre le français de Saint-Henri, quartier populaire de Montréal, dans la première moitié du $\mathrm{xx}^{\mathrm{e}}$ siècle, et le français général de la même époque?

\section{CONCLUSION}

La traduction est la pierre de touche de toute théorie linguistique ayant la prétention d'expliquer, dans le cadre d'une théorie générale des signes, les propriétés spécifiques de nos langues naturelles. Nous avons vu quelles sont les principales qualités qui les distinguent de tout système d'information construit logiquement, mathématiquement, de toute langue technologique. Ayant subi, à travers les âges, les influences les plus disparates, portant l'empreinte d'innombrables facteurs hétérogènes, chacune de nos langues est un ensemble de formes et de structures instrumentales pleines d'incohérence, et de contradictions, ce que les grammairiens du Grand Siècle appelaient «les caprices» ou « les bizarreries de l'usage ». C'est dire que toutes ces formes et ces structures instrumentales ne sont pas, ne peuvent pas être motivées par l'univers mental qu'elles symbolisent, par les formes et les structures du monde qui nous entoure, du monde qui est en nous ; dans une très large mesure elles sont arbitraires, ou conventionnelles. Traduire, c'est découvrir sous cette surface instrumentale la structure mentale sous-jacente, par exemple sous la périphrase verbale française il vient d'arriver la structure mentale "passé récent», pour remonter ensuite de cette structure mentale à la surface instrumentale du registre adverbial anglais : he's just arrived. Traduire, ce n'est pas remplacer un instrument par un autre instrument; c'est trouver, sous les surfaces instrumentales de deux langues différentes, la forme et la structure mentale communes. Les limites de la traduction au sens strict cö̈ncident très exactement avec celles de ce commun dénominateur mental.

Le traducteur est le premier explorateur de ce monde intérieur des formes et des structures mentales. Le traducteur est le meilleur guide du linguiste comparatiste, et toute linguistique digne de ce nom est comparaison. L'observation attentive, l'analyse méthodique des traductions aujourd'hui à notre disposition en nombre infini, nous permettent de saisir nos langues dans leur réalité vivante, avec toutes leurs qualités et tous leurs défauts, nous montrant dans leurs véritables proportions les servitudes et les grandeurs du langage humain.

MARIO WANDRUSZKA 


\section{QUESTIONS}

N... : En ce qui concerne les structures mentales, est-ce que, par exemple, le passif en anglais correspond tout simplement au passif en français?

Réponse : C'est tout d'abord un problème instrumental. On peut traduire dans la mesure où il $\mathrm{y}$ a un dénominateur commun; reste à savoir, et c'est évidemment un vaste sujet, dans quelle mesure les structures mentales sont différentes. Si elles étaient profondément différentes, on ne pourrait plus traduire, parce qu'il reste toujours quelque chose d'intraduisible, c'est-à-dire que nous vivons, en nous exprimant par exemple en anglais, dans un univers mental incompréhensible à des hommes s'exprimant à l'aide d'un autre instrument. Il reste donc évidemment toujours quelque chose d'intraduisible, mais en bonne méthode nous devons tout d'abord comparer les possibilités de traduction, c'est-à-dire les structures instrumentales qui ont ce dénominateur commun mental. L'école idéaliste a voulu interpréter toutes les structures instrumentales comme autant de structures mentales différentes, c'est-à-dire que les trois mille langues parlées de par le monde seraient autant d'univers mentaux différents, sans possibilité de contact, sans possibilité de compréhension. Or, il y a tout de même quelque chose d'universel, c'est pourquoi aujourd'hui la linguistique se penche à nouveau avec un intérêt accru sur le problème des universaux. Il y a dans la voie passive une structure mentale, incontestablement ; on peut en effet parler d'un sujet qui est en même temps objet d'une action, d'un processus sans que l'on nomme, sans que l'on puisse nommer, sans que l'on veuille nommer l'agent : You are wanted on the phone, On vous demande au téléphone. Est-ce vraiment une structure mentale différente? Est-ce que les anglophones vivent par là même dans un univers mental légèrement différent parce qu'ils voient tout sous cet angle-là ? J'ai répondu à votre question dans un article où je compare, mais là en partant des structures instrumentales, plusieurs langues. Il y a aussi il ne faut pas l'oublier la voie pronominale : English spoken, On parle français, Si parla italiano. Est-ce que c'est une structure mentale? Qu'est-ce que c'est comme structure mentale? Se habla espanol. C'est un instrumen et pas autre chose. Il y a certainement quelque chose de mental en profondeur, mais tout d'abord il faut comparer pour qu'on puisse parler de traduction.

M. Darbelnet : Le passif étant plus fréquent en anglais qu'en français pour des raisons de structure, du fait, pour ne prendre qu'un exemple, que l'on peut fabriquer des passifs avec des verbes intransitifs, ce qui n'est pas possible en français. Il y a là une facilité pour l'expression qui peut être mise à contribution, qui peut créer certains besoins. Il y a une certaine réserve, une certaine circonspection anglaise dans les discussions qui s'accommodent très bien d'un passif qui permet de glisser sur l'origine d'une proposition, de la présenter comme un fait et ceci est en accord avec la phénoménalité de l'anglais. La question du résidu intraduisible se présente dans les mots de culture ou les mots de civilisation. Pour prendre un exemple très simple, on ne peut pas refuser l'équivalence ou l'équation bread et "pain". Il est impossible de traduire "pain" autrement que par bread, dans la plupart des contextes en tout cas. Mais il est bien évident que le pain anglais et le pain français, ce n'est pas le même objet et que cet objet différent au départ n'occupe pas la même place dans chacune des deux cultures. Réponse : Je vous rappelle l'admirable essai d'Ortega y Gasset Miseria y esplendor de la traducción où il dit : comment voulez-vous traduire le mot allemand Wald en espagnol puisqu'il n'y a pas de Wald en Espagne? $E l$ bosque, ce n'est pas la forêt germanique, donc les structures mentales, l'univers mental dans notre for intérieur est différent bien entendu, mais ceci est évidemment la dernière conséquence que les structures instrumentales des langues font apparaître.

M. Tréveau : Vous avez parlé des variations et des constantes dans la langue. Il y a un phénomène dans la littérature nord-américaine actuellement, c'est le phénomène hippie, qui a quand même une très forte influence sur la littérature et sur d'autres phénomènes en Amérique du Nord. Je pense particulièrement aux auteurs comme Timothy Leary et Alan Ginsberg, qui ont plus ou moins inventé ce qu'ils ont appelé la sub-culture. C'est non seulement une invention culturelle, mais c'est aussi une invention linguistique. Je suis tombé sur une traduction de Timothy Leary où il $y$ avait un affaiblissement considérable de la teneur du message original. Les schèmes de pensée, par exemple, étaient complètement différents en français et en anglais. Que 
deviendra l'univers mental du français ou des langues romanes en général lorsqu'on arrivera aux schèmes de pensée de la littérature nord-américaine, de la nouvelle littérature nord-américaine ? Est-ce que vous pensez qu'on arrivera à trouver des équivalences fondamentales dans l'univers mental français, par exemple, ou bien qu'on sera condamné, comme on l'est actuellement, à faire des emprunts à l'anglais et à franciser tous ces termes nouveaux?

Réponse : Je crois que dans votre question il y a deux choses : a) Le facteur instrumental, c'est-à-dire les particules verbales de l'anglais qui sont admirables (drop out, teach in, love in, etc.) et qu'on ne peut pas traduire parce que c'est l'instrument qui fait défaut dans les langues romanes. Comme dans ces langues l'instrument n'existe pas, on prend le mot tel quel et on s'en sert parce qu'il n'y a pas d'équivalence, il n'y a pas d'équivalence formelle aussi handy, aussi praticable. Par conséquent, c'est un aspect instrumental ; $b$ ) Le rapport exact entre la variante et la constante est absolument intraduisible. Il y a certaines connotations dans stoned, connotations qui n'existent évidemment que par rapport à la langue normale, à la langue reçe. Ce rapport-là, il faudrait le réinventer, il faudrait trouver quelque chose d'à peu près analogue, d'à peu près équivalent, et à ce moment il ne s'agit plus de traduction, mais de réinvention dans le cadre des possibilités françaises. Je ne veux pas évidemment me faire l'avocat de l'anglicisme, mais on comprend pourquoi tant de mots ayant un sens très précis dans le contexte de la langue, des constantes, comme des variantes de subculture, des variantes hippies, sont pris et tout simplement introduits dans un contexte français, italien, allemand ou russe, parce qu'on renonce à la traduction. Il ne s'agit pas seulement d'ailleurs de culture hippie et je n'en veux pour preuve que design. C'est un mot qui vient du français n'est-ce pas, « dessin »? Une grande partie du lexique anglais, c'est de l'ancien français qui a mal tourné ! On a donc design et on sait exactement ce que cela veut dire. D'ailleurs il n'y a pas de définition qui vaille. Tout le monde, en Allemagne, en Italie, partout, dit maintenant design.

Pour le français, c'est le problème instrumental qui est plus grave parce qu'il y a la graphie aberrante, il y a la prononciation. On a besoin de ce mot-là et on ne peut pas le digérer, on ne peut pas l'intégrer complètement, par conséquent, il y a une certaine tension, qui gêne jusqu'au jour où on aura trouvé un mot bien français pour dire la même chose. 